\title{
ESTUDIO PALEOAMBIENTAL DE LOS DEPÓSITOS LITORALES SOMEROS DEL PLIOCENO INFERIOR DE FUERTEVENTURA (ISLAS CANARIAS)
}

\author{
Esther MARTÍN GONZÁLEZ', Carolina CASTILLO', \\ Margarita GUTIÉRREZ GONZÁLEZ² \\ y Julio AGUIRRE
}
' Dpto. de Biología Animal, Fac. de Biología. Universidad de La Laguna. Avda. Astrofísico Sánchez, s/n. 38206 La Laguna, Tenerife. e-mail: ccrui@ull.es
? Dpto. de Edafología y Geología, Fac. de Biologİía. Universidad de La Laguna. Avda. Astrofísico Sánchez, s/n. 38206 La Laguna. Tenerife.
'Dpto. de Estratigrafía y Paleontología, Fac. de Ciencias. Universidad de Granada. Campus de Fuentenueva, s/n. 18071 Granada. e-mail: jaguirre@goliat.ugr.es

Martín González, E., Castillo, C., Gutiérrez González, M. y Aguirre, J. 2001. Estudio paleoambiental de los depósitos litorales someros del Plioceno inferior de Fuerteventura (Islas Canarias). [Palaeoenvironmental study of the shallow coastal deposits of the Lower Pliocene of Fuerteventura (Canary Islands).] Revista Española de Paleontología, n. ${ }^{\circ}$ extraordinario, 47-57. ISSN 0213-6937.

\begin{abstract}
Shallow littoral deposits, belonging to the Early Pliocene, are continuously exposed along ca. $40 \mathrm{~km}$ of the western coast of Fuerteventura Island (Canary Islands). The studied materials constitute a terrigenous sequence made up of fossiliferous sands, breccias and conglomerates deposited in a fan-delta. The sediments were flowed out in very shallow nearshore settings, probably in the coastal zone, and were only slightly modified by marine processes (wave action, storm waves, tidal currents, etc.). Faunal composition, mainly represented by patelloid gastropods, oysters and balanids, is consistent with a shallow littoral setting. The studied Pliocene sediments are vertically arranged constituting a deepening-shallowing cycle. Fossil remains show clear evidence of strong taphonomic destruction (abrasion, corrasion, fragmentation, facets, disarticulation, and so on). These taphonomic traits also agree with a shallow origin of the deposits, where highly hydraulic energy leads to this sort of preservation. This allows, in turn, to infer that the deposition was dominated by background biostratinomic processes (background-dominated sequences according to Speyer and Brett, 1991). Nonetheless, several pieces of evidence suggest that horizontal transport of fossils was no important, since sudden catastrophic events of sediment discharges due to the progradation of the fan delta buried the organisms: a) the preserved fauna is consistent with the type of substrate; b) the low representation of borings; c) the angulosity of boulders and cobbles, and the low sorting; and, d) preferred concave-up orientation of shells. Presence of species inhabiting present-day tropical-subtropical environments in the Pliocene sediments of Fuerteventura strongly suggests a northwards shifting of the tropical bands during higher global temperature at that time.
\end{abstract}

Keywords: Coastal deposits, taphonomy, palaeoecology, Early Pliocene, Canary Islands.

\section{RESUMEN}

A lo largo de unos $40 \mathrm{~km}$ de la costa occidental de la isla de Fuerteventura (Islas Canarias) afloran unos depósitos litorales de edad Plioceno inferior. Los materiales estudiados constituyen una secuencia terrígena formada por arenas, brechas y conglomerados fosilíferos depositados en un ambiente de abanico deltaico. El material detrítico aportado a la cuenca fue depositado en las zonas cercanas a la costa, probablemente en la misma franja litoral, donde apenas sufrió modificaciones por los procesos marinos (acción del oleaje, oleaje de tormentas, corrientes mareales, etc.). La fauna que contiene, fundamentalmente gasterópodos pateliformes, ostreidos y cirrípedos, es coherente con un medio litoral somero. Los materiales se ordenan en la vertical formando un ciclo de profundización-somerización completo. Los restos fósiles contenidos muestran evidencias de haber estado sometidos a un alto grado de destrucción tafonómica (abrasión, fragmentación, desarrollo de facetas, desarticulación, etc.). Estos rasgos tafonómicos son congruentes con un medio litoral somero, donde la 
alta energía hidraúlica condiciona fuertemente el tipo de conservación. Esto permite inferir que durante el depósito de los materiales estudiados dominaron los procesos tafonómicos que acontecen durante los periodos normales de sedimentación ("background-dominated sequences" según Speyer y Brett, 1991). No obstante, una serie de criterios indican que el transporte en la horizontal no debió ser importante, ya que se produjeron enterramientos catastróficos repentinos debido a los continuos aportes de material siliciclástico desde el abanico deltaico: a) la congruencia entre el tipo de fauna y el tipo de substrato; b) la escasez de bioperforaciones; c) la angulosidad de los cantos y la escasa selección por tamaños; y, d) la orientación preferencial de conchas con la concavidad hacia arriba. La presencia en los yacimientos del Plioceno de Fuerteventura de algunas especies con una distribución tropical-subtropical en la actualidad, justifica la idea de un desplazamiento latitudinal hacia el norte de las bandas tropicales durante esta época en condiciones globales de temperaturas más elevadas.

Palabras clave: Depósitos litorales, tafonomía, paleoecología, Plioceno inferior, Islas Canarias.

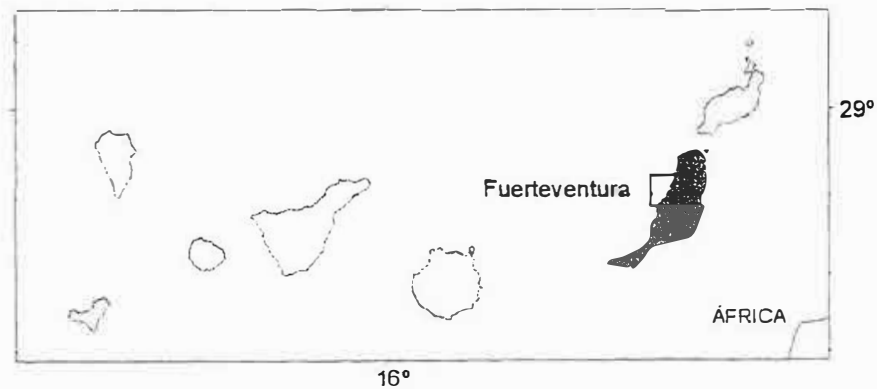

$29^{\circ}$

옹

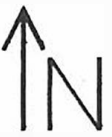

Complejo Basal
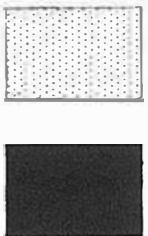

Coladas basálticas Pliocenas (Serie II)

Formaciones sedimentarias pliocuatemarias

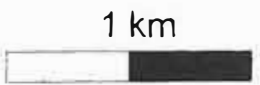

Playa del Valle

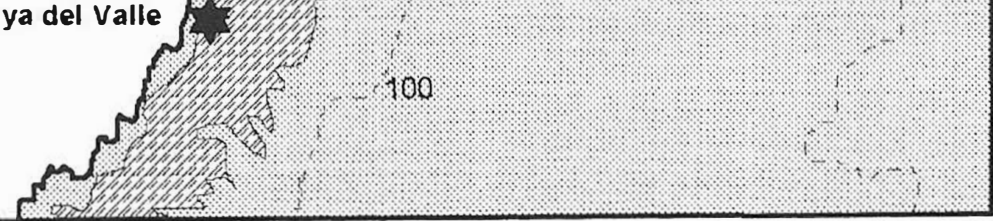

3158000

Figura 1. Mapa geológico simplificado de la zona de estudio, mostrando la localización de los yacimientos de Playa del Valle y Barranco León.

Geological sketch of the studied area showing the location of the paleontological sites at Playa del Valle and Barranco León. 


\section{INTRODUCCIÓN}

Tradicionalmente se ha prestado escasa atención a las asociaciones de organismos que colonizaron los ambientes litorales, fundamentalmente porque se trata de ambientes donde predomina la erosión frente a la sedimentación debido a la alta turbulencia hidráulica del medio (Johnson, 1988, 1992; Johnson y Baarli, 1999). No obstante, desde la primera recopilación de datos sobre las paleocomunidades de ambientes litorales rocosos realizada por Johnson (1992) se han sucedido una serie de trabajos que han incrementado dicha base de datos, tanto sobre los restos de organismos fósiles encontrados en estos medios (Johnson y Baarli, 1999) como sobre las trazas fósiles debidas a su actividad (Martinell y Domènech, 1995; Gibert et al., 1996, 1998; Silva et al., 1999).

Un factor importante a tener en cuenta que favorece la conservación de las comunidades litorales es la existencia de una elevada tasa de sedimentación o de eventos de enterramientos catastróficos, especialmente en ambientes costeros próximos a la desembocadura de un sistema fluvial. Debido al continuo aporte de material terrígeno los organismos pueden ser enterrados rápidamente y permanecer aislados de los procesos bioestratinómicos que tienen lugar en la zona tafonómicamente activa (TAZ sensu Powell et al., 1989). De esta forma, el potencial de preservación de estas comunidades se ve incrementado (Powell et al., 1989; Kidwell y Bosence, 1991; Aguirre y Jiménez, 1997, 1998).

La utilización conjunta de criterios estratigráficos, sedimentológicos, y tafonómicos ayuda a inferir las condiciones paleoambientales y la dinámica sedimentaria de estos medios costeros alimentados por material terrígeno. En última instancia, son herramientas necesarias y de gran valor para establecer modelos de evolución secuencial.

A lo largo de la costa occidental de la isla de Fuerteventura (Islas Canarias) aflora un conjunto de materiales depositados en ambientes litorales someros alimentados por depósitos siliciclásticos. Constituyen una secuencia terrígena formada por brechas y conglomerados fosilíferos, polimícticos y heterométricos, entre los que se intercalan algunos bancos de arenas y niveles de acumulación de fósiles (Meco, 1975; Meco y Stearns, 1981).

En este trabajo presentamos un modelo de evolución paleoambiental y de evolución secuencial inferido a partir del estudio de las facies y de los patrones de conservación de los fósiles contenidos en estos depósitos. Por ello, este estudio contribuye a la ampliación de la nueva base de datos recientemente publicada por Johnson y Baarli (1999) que recopila datos sobre las paleocomunidades que habitaron los ambientes costeros.

\section{METODOLOGÍA}

Se han estudiado tres secciones situadas en dos áreas de la franja costera occidental de Fuerteventura. La

\section{Columna 1}

Columna 2

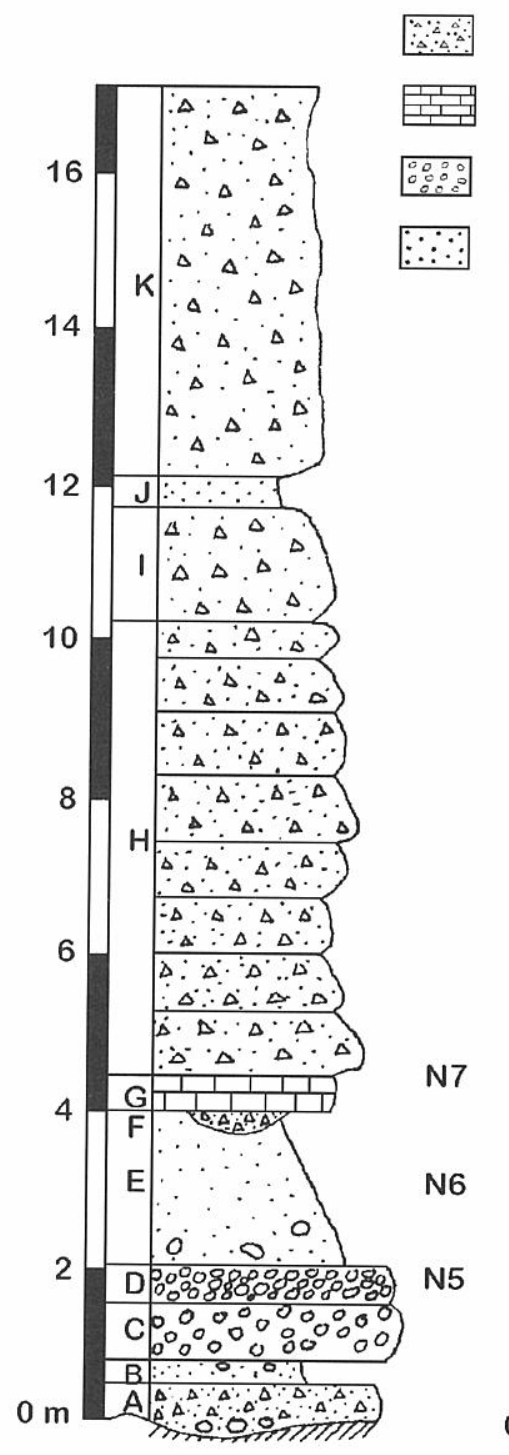

1

2

3

4

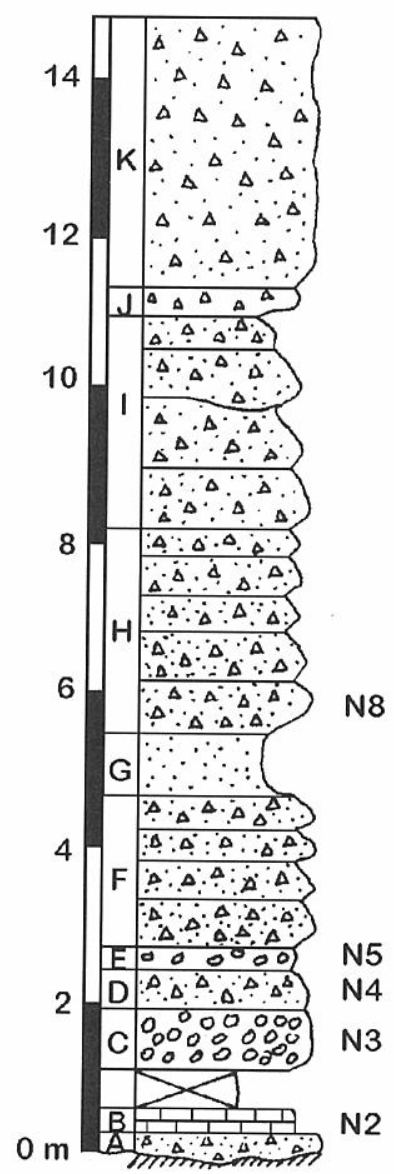

Figura 2. Columnas estratigráficas de Playa del Valle. 1. Brechas polimícticas; 2. Nivel bioclástico cementado; 3. Conglomerados; 4. Arenas. Los niveles fosilíferos vienen indicados en negrita (N1 a N8).

Stratigraphic columns of Playa del Valle. 1. Breccias; 2. Cemented bioclastic bed; 3 . Conglomerates; 4. Sands. Fossiliferous beds are indicated in boldface (N1 to N8).

primera zona se localiza en la Playa del Valle, aproximadamente $200 \mathrm{~m}$ al S de la localidad homónima, y la segunda en Barranco León, localidad situada unos 6 $\mathrm{km}$ al S del Puerto de los Molinos (Fig. 1). En el primer afloramiento la sección se expone en un transecto N-S de aproximadamente $100 \mathrm{~m}$, en el cual se pueden observar las relaciones laterales de facies y se pueden seguir lateralmente los diferentes niveles fosilíferos en continuidad estratigráfica. Aquí, debido a la existencia de rápidos cambios laterales de facies y de acuñamientos laterales se han levantado dos columnas estratigráficas, 


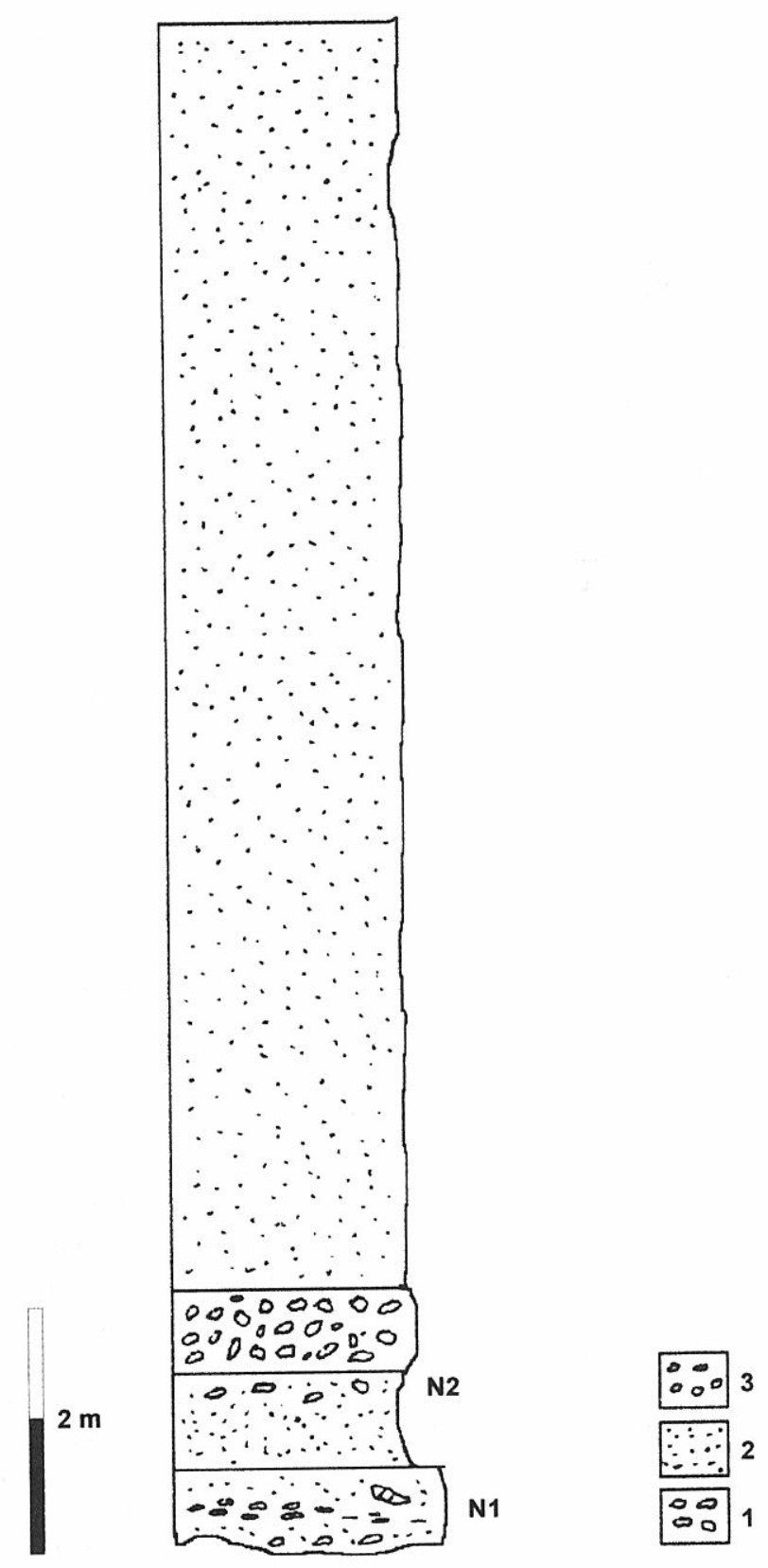

Figura 3. Columna estratigráfica de Barranco León. 1. Brechas polimícticas; 2. Arenas; 3. Conglomerados. Los niveles fosilíferos se indican en negrita (N1, N2).

Stratigraphic column of Barranco León. 1. Breccias; 2. Sands; 3. Conglomerates. Fossiliferous beds are indicated in boldface ( $N-1$, N2).

separadas unos 75 m (Fig. 2). En Barranco León sólo se ha levantado una columna estratigráfica (Fig. 3).

En las secciones levantadas en Playa del Valle se han reconocido hasta ocho niveles fosilíferos (N-1 a N-8 en la Fig. 2), mientras que en la sección Barranco León sólo dos (N-1 y N-2 en la Fig. 3). En todos los niveles de ambos afloramientos se hicieron observaciones cualitativas sobre diversos atributos tafonómicos, como grado de desgaste, orientación, fragmentación, articulación, tipo de empaquetamiento, presencia de facetas, etc. Para completar estos datos cualitativos observables en el afloramiento se realizaron medidas de algunos de estos atributos tafonómicos en tres niveles fosilíferos de Playa del Valle (N-1, N-4 y N-6) y en el segundo nivel de Barranco León (N-2). En los niveles de Playa del Valle se midieron: a) proporción de conchas completas frente a fragmentadas, b) orientación con respecto a la concavidad de las conchas, y, c) proporción de conchas con ordenamiento empaquetado o encajado ("stacking" o "nesting" respectivamente según Kidwell et al., 1986). Estas medidas se realizaron en una superficie de $1 \mathrm{~m}^{2}$ en cada uno de los niveles muestreados.

En el caso de Barranco León, sólo se realizaron medidas sobre la orientación de las conchas con respecto a la concavidad y con respecto al plano de estratificación (ángulo mínimo que forman las conchas con la superficie de estratificación), con objeto de conocer su ordenamiento tridimensional. Aquí, las medidas se realizaron en diez estaciones de muestreo de aproximadamente $1 \mathrm{~m}^{2}$ cada una ( $1 \mathrm{~m}$ de lado por $0,85 \mathrm{~m}$ de alto — potencia del nivel fosilífero-).

\section{CONTEXTO GEOLÓGICO Y DESCRIPCIÓN ESTRATIGRÁFICA}

Los sedimentos estudiados se disponen discordantemente sobre los materiales volcánicos del Complejo Basal, fosilizando una plataforma de abrasión situada entre 10 y 20 $\mathrm{m}$ sobre el nivel del mar. Por encima, se disponen unos sedimentos aluviales y de dunas calcareníticas que, localmente, pueden alcanzar hasta $30 \mathrm{~m}$ de espesor (Meco, 1975). Según este autor, los materiales aluviales son interpretados como sedimentos formados en condiciones más húmedas (más pluviosidad) que las dunas, que corresponden a depósitos de un régimen más árido.

Meco (1975, 1977), basándose en el contenido macrofaunístico, asignó una edad de tránsito MiocenoPlioceno al conjunto terrígeno estudiado en este trabajo. Así mismo, resaltó la extraordinaria abundancia y riqueza específica de organismos intertropicales, como Strombus coronatus Defrance, Nerita emiliana Mayer, Gryphaea virleti Deshayes (= Saccostrea cuccullata Born) y Rothpletzia rudista Simonelli.

La asignación temporal de estos materiales establecida por Meco $(1975,1977)$ ha sido confirmada mediante dataciones radiométricas con el método K-Ar. Las dataciones realizadas en coladas volcánicas de la Serie II-A sensu Fúster et al. (1968), contemporáneas con los niveles de arenas que se encuentran en la base de la secuencia de la Playa del Valle, indican una edad de 5,8 \pm 0,5 Ma según Meco y Stearns (1981) o de 5,0 \pm 0,3 Ma según Coello et al. (1992). En otras localidades de la isla se encuentran coladas de la Serie II-B por encima de los depósitos aluviales y de las dunas calcareníticas que cubren los materiales marinos. Estas coladas volcánicas han proporcionado edades por debajo de los 3 Ma (Coello et al., 1992). Por tanto, la unidad marina estudiada en este trabajo tiene un rango temporal máximo, entre 5,8 y $3 \mathrm{Ma}$; es decir, desde el Messiniense terminal hasta la base del Plioceno superior. 


\section{SECCIÓN PLAYA DEL VALLE}

En este afloramiento la secuencia pliocena estudiada comienza con un banco conglomerático que rellena las irregularidades erosivas creadas en el substrato volcánico (Complejo Basal). Se trata de unas brechas polimícticas con fragmentos angulosos de cantos de gran tamaño procedentes del Complejo Basal, que contiene intercalaciones de cantos redondeados de 0,5-1 cm de tamaño medio. Así mismo, en este tramo se encuentran algunas concentraciones discontinuas de bioclásticos muy cementadas con una coloración rosácea (N-1 en la Fig. 2). Inmediatamente por encima del nivel fosilífero $\mathrm{N}-1$ se encuentra otra concentración bioclástica más o menos continua que alcanza hasta $0,5 \mathrm{~m}$ de espesor (N-2 en la Fig. 2). Esta parte baja de la secuencia está caracterizada por un dispositivo geométrico de solapamiento expansivo.

Por encima, la secuencia continúa con un conjunto de brechas y conglomerados clastosoportados, con cantos fundamentalmente basálticos y poco redondeados, y con una matriz tamaño arena media-gruesa. Las brechas y los conglomerados suelen presentar muros irregulares y erosivos, y contienen tres niveles fosilíferos (N-3, N-4 y N-5 en la Fig. 2).

En la parte media-inferior de la sección hay un banco de $1 \mathrm{~m}$ de espesor de arenas medias que disminuye de tamaño de grano hacia el S hasta arenas finas (Columna II de la Fig. 2). En la base de este nivel aparecen cantos de 1 a $5 \mathrm{~cm}$ de diámetro máximo, variando entre subangulosos a subredondeados, principalmente de composición basáltica. Contiene abundantes restos fósiles, que aparecen dispersos entre las arenas o concentrados en pequeños paleocanales en el techo de este tramo arenoso, y que se corresponden con el nivel fosilífero N-6 (Fig. 2). Por encima de estas arenas se encuentra un nivel bioclástico muy cementado compuesto por una gran cantidad de conchas y moldes de moluscos de pequeño tamaño, con menor proporción de fragmentos basálticos, que se corresponde con el nivel N-7 (Fig. 2).

Por encima, la secuencia pliocena sigue hasta el techo con unos 10-15 m de potencia de paquetes de brechas polimícticas compuestas por cantos heterométricos poco seleccionados incluidos en una matriz arenosomicroconglomerática. Se trata de niveles de brechas y conglomerados con bases canalizadas y un ordenamiento interno caracterizado por una selección positiva de tamaños de grano. Los cantos siguen siendo de naturaleza volcánica, principalmente basálticos, y subredondeados. Estos niveles contienen concentraciones fosilíferas en capas más o menos continuas en la horizontal; en la base de este conjunto brechoide se encuentra el nivel fosilífero N-8 (Fig. 2).

\section{SECCIÓN BARRANCO LEÓN}

La sección Barranco León muestra rasgos sedimentológicos más homogéneos y monótonos que la sección Playa del Valle. En la base aflora un conglomerado de tamaño grava muy bien seleccionado compuesto por clastos redondeados, y cuyo espesor es de unos $55 \mathrm{~cm}$ (Fig. 3). Asociados a estas gravas aparecen niveles carbonatados discontinuos muy cementados y ricos en fósiles, principalmente bivalvos (N-1 en la Fig. 3). Este nivel engloba algunos cantos de diques muy grandes del Complejo
Basal que debían estar en la plataforma cuando se estaban depositando las arenas.

Por encima se observa un nivel de arenas de color amarillo con cantos que muestra una clara granoselección negativa. Los cantos que aparecen en el techo de este tramo arenoso son redondeados y alcanzan hasta $10 \mathrm{~cm}$. En esta parte superior se encuentra un nivel muy rico en fósiles (N-2 en la Fig. 3). A continuación hay un conglomerado polimíctico clastosoportado, con cantos de diferente composición y tamaño, englobados en una matriz arenosa. Este conglomerado contiene fragmentos dispersos de conchas.

Superponiéndose a los conglomerados anteriores, y hasta el techo de la secuencia pliocena, aflora un tramo de arenas eólicas, de 10 a 12 m de espesor, con estratificación planar y cruzada.

\section{COMPOSICIÓN TAXONÓMICA Y ASPECTOS TAFONÓMICOS}

Tanto en Playa del Valle como en Barranco León, la composición taxonómica de las asociaciones de organismos y el grado de conservación de los fósiles son muy similares. Por ello, en la descripción que se realiza a continuación se hará referencia a los rasgos comunes de ambas secciones, especificando las características distintivas observadas en la sección correspondiente. Las asociaciones de fósiles en ambos afloramientos son poco diversas, estando dominadas principalmente por moluscos (bivalvos y gasterópodos) y cirrípedos (balánidos).

En general, predominan los fósiles fragmentados (Tabla 1) y con las conchas parcial o totalmente desgastadas por abrasión o "corrasión" (según Brett y Baird, 1986). En relación con la disposición de las conchas con respecto a la concavidad, más del $70 \%$ de las conchas del segundo nivel fosilífero de Barranco León se disponen con la concavidad hacia abajo (Tabla 1). Por el contrario, en los niveles de Playa del Valle dominan los restos orientados preferencialmente con la concavidad hacia arriba (Tabla 1), que es una posición hidrodinámicamente inestable. En cuanto a la disposición de los fósiles con respecto a la estratificación, rasgo medido exclusivamente en Barranco León, éstos se orientan mayoritariamente de forma concordante; es decir, con un ángulo inferior a $30^{\circ}$ (Tabla 1). El grado de empaquetamiento y la concentración de fósiles (terminología sensu Kidwell y Holland, 1991) en los niveles de acumulación es muy variable, oscilando entre acumulaciones poco densas, con una concentración del $5 \%$ de restos fósiles por volumen de roca, hasta acumulaciones densas, con más del 30-40\%. Cabe destacar, así mismo, la notable escasez de trazas de bioperforaciones, representadas por los icnogéneros Entobia y Gastrochaenolites. También se observan algunas conchas con unas marcas superficiales que consisten en pequeñas galerías espirales de una única vuelta de espira. Pueden atribuirse a las marcas dejadas por organismos epibiontes, probablemente gasterópodos vermétidos. 


\begin{tabular}{|c|c|c|c|}
\hline \multicolumn{4}{|c|}{ Playa del Valle } \\
\hline & $\begin{array}{c}\text { Nivel } 1 \\
(63)\end{array}$ & $\begin{array}{l}\text { Nivel } 4 \\
(92)\end{array}$ & $\begin{array}{c}\text { Nivel } 6 \\
(105)\end{array}$ \\
\hline Completos & $10,2 \%$ & $47,1 \%$ & $38,6 \%$ \\
\hline Fragmentos & $89,8 \%$ & $52,9 \%$ & $63,2 \%$ \\
\hline Concavidad abajo & & $37,9 \%$ & $23,8 \%$ \\
\hline Concavidad arriba & & $62,1 \%$ & $76,2 \%$ \\
\hline Empaquetamiento & & & $10,5 \%$ \\
\hline Articulación ${ }^{*}$ & $0 \%$ & $0 \%$ & $19 \%$ \\
\hline Valvas derechas* & & $70,3 \%$ & $79,2 \%$ \\
\hline Valvas izquierdas* & & $29,7 \%$ & $20,8 \%$ \\
\hline \multicolumn{4}{|c|}{ Barranco León } \\
\hline & $\begin{array}{c}\text { Nivel } 2 \\
(494)\end{array}$ & & \\
\hline Concavidad abajo & $79,6 \%$ & & \\
\hline Concavidad arriba & $20,4 \%$ & & \\
\hline Angulo $<30^{\circ}$ & $81,2 \%$ & & \\
\hline Angulo $30^{\circ}-60^{\circ}$ & $5,7 \%$ & & \\
\hline Angulo $>60^{\circ}$ & $13,1 \%$ & & \\
\hline Empaquetamiento & $6,1 \%$ & & \\
\hline
\end{tabular}

Tabla 1. Atributos tafonómicos correspondientes a las secciones de Playa del Valle y Barranco León. Los porcentajes se han obtenido a partir de los fósiles más abundantes en todos los niveles de los dos afloramientos: gasterópodos, ostreidos y balánidos. Entre paréntesis se indica el número de bioclastos por unidad de superficie medida (ver apartado de Metodología). El asterisco indica que para el cálculo del porcentaje se han tenido en cuenta exclusivamente los ostreidos. Estos valores complementan las observaciones realizadas en ambos afloramientos.

Taphonomic traits in the Playa del Valle and Barranco León sections. The percentages have been estimated taken into account the more abundant fossils in both sections: gastropods, ostreids and barnacles. The number of bioclasts per unit of surface is indicated in brackets. For taphonomic traits followed by an asterisk only ostreids have been considered. These values complete the observations carried out in both sections.

Los organismos más abundantes y mejor representados en los afloramientos son los gasterópodos pateliformes de conchas calcíticas. Ocasionalmente, estos organismos forman concentraciones pauciespecíficas (terminología según Kidwell et al., 1986), casi exlusivamente representadas por acumulaciones de sus conchas. La especie más común en prácticamente todos los niveles fosilíferos es Patella cf. caerulea Linné (Subclase Eogastropoda, Orden Patellogastropoda, Familia Patellidae). Esquema de clasificación de Beesley et al., 1998). Son formas de gran tamaño (hasta $9 \mathrm{~cm}$ de diámetro) que se encuentran actualmente en los archipiélagos de Azores y Madeira y en el Mediterráneo. Además de estos pateloides, también se encuentran Diodora cf. gracea (Linné) y la especie caribeña
Fisurella cf. rosea (Gmelin). Aunque morfológicamente son muy similares a los pateloides, Beesley et al. (1998) incluyen estos dos géneros en la familia Fissurellidae (Subclase Orthogastropoda, Superorden Vetigastropoda).

Los gasterópodos pateliformes (término que incluye los representantes de ambas familias) aparecen con las conchas conservadas, generalmente fragmentadas y, tanto la ornamentación como las aristas, muestran evidencias de desgaste y redondeamiento. No obstante, en el afloramiento Barranco León es más frecuente la existencia de individuos poco desgastados por abrasión. Otro atributo tafonómico muy conspicuo de los gasterópodos pateliformes en los materiales estudiados es la existencia de facetas en la zona apical de la concha, lo que dificulta (e impide, en numerosos casos) la determinación de las especies, e incluso de los géneros. Ocasionalmente, se encuentran individuos completos no desgastados, que permiten su identificación. En algunos niveles fosilíferos (por ejemplo, en los niveles N-5 y N-6 de Playa del Valle, Fig. 2) es frecuente que las conchas de estos gasterópodos presenten un ordenamiento empaquetado y/o encajado (Tabla 1).

En orden de abundancia, los cirrípedos constituyen el segundo grupo de organismos más abundante en las asociaciones de fósiles. Los componentes de este grupo no han podido ser determinados, aunque cabe destacar la presencia de varias especies. Es interesante señalar, entre ellas, la existencia de una especie perteneciente al género Tetraclita, cirrípedo que ocupa en la actualidad la zona tropical de California, el Golfo de Méjico y la región panameña (Zullo, 1986, 1991).

Usualmente, los balánidos no aparecen formando concentraciones, sino que se encuentran dispersos, tanto entre los cantos volcánicos como entre los restos bioclásticos que constituyen los niveles de acumulación. Dominan las conchas fragmentadas frente a las completas, las cuales suelen estar desplazadas de su posición de crecimiento. Es frecuente, además, encontrar ejemplares sin la zona apical conservada y con claras evidencias de desgaste de las conchas por abrasión y/o "corrasión".

Además de las especies de gasterópodos pateliformes anteriormente descritas, también se han identificado algunos representantes de las familias Naticidae (Superorden Caenogastropoda) (e.g. Natica sp.) y Neritidae (Superorden Neritopsina), como Nerita emiliana Mayer (clasificación supragenérica según Beesley et al., 1998). En todos los casos se conservan como moldes ya que son formas con concha originalmente aragonítica. No obstante, ocasionalmente se encuentran algunos individuos que conservan restos de la concha original. El hecho de preservarse mayoritariamente como moldes dificulta su identificación a nivel específico e incluso genérico.

Entre los bivalvos, son muy abundantes las valvas de Saccostrea cuccullata Born, ostreido típico de lagunas costeras y manglares tropicales-subtropicales actuales (Stenzel, 1971; Harry, 1985; Okemwa et al., 1986; Morton y Chan, 1986) y fósiles (Aguirre y Jiménez, 1994; Aguirre, 1998). En concreto, son especialmente 
abundantes en los niveles N-4 y N-6 de Playa del Valle. En la inmensa mayoría de los casos las valvas aparecen desarticuladas, con valores que oscilan entre el $81 \%$ del nivel N-6 y el $100 \%$ de los niveles N-1 y N-4 de Playa del Valle (Tabla 1). Así mismo, las conchas están muy redondeadas, con el margen y la ornamentación desgastados, debido, probablemente, a procesos de abrasión mecánica. Al igual que en los gasterópodos pateliformes, las valvas de Saccostrea muestran facetas de desgaste en la zona del umbo. En cuanto a la orientación de las valvas con respecto a la concavidad, más del $70 \%$ de las valvas están con la concavidad hacia abajo. Por otro lado, también se reconoce un sesgo en la conservación según el tipo de valva, predominando las valvas derechas sobre las izquierdas (más del 70\% son valvas derechas) (Tabla 1 ).

Además de $S$. cuccullata, en el primer nivel de acumulación de Barranco León se han reconocido conchas del género Chlamys, algunas de los cuales están incluso articuladas. También se encuentran moldes de bivalvos de pequeño tamaño, que en muchos casos están muy fragmentados, lo que imposibilita su determinación. Se han podido identificar formas infaunicas someras de las familias Cardiidae y Veneridae (Orden Heterodonta según Beesley et al., 1998). La presencia de estos moldes es especialmente importante en el nivel fosilífero N-8 de Playa del Valle. Esto hace que sea un nivel rico y con mayor diversidad faunística que los demás donde se han conservado mayoritariamente los restos calcíticos (gasterópodos pateliformes, ostreidos y balánidos). En dicho nivel, asociadas con la fauna, aparecen algas coralináceas formando rodolitos de reducidas dimensiones.

\section{DISCUSIÓN}

\section{INTERPRETACIÓN PALEOAMBIENTAL E HISTORIA TAFONÓMICA}

Los sedimentos pliocenos de Playa del Valle se interpretan como un conjunto de materiales depositados en un ambiente de abanico deltaico. La fauna que caracteriza estos materiales (gasterópodos pateliformes, cirrípedos, natícidos y ostreidos), a su vez, indica que la descarga del sedimento tuvo lugar cerca de la costa, probablemente en la zona submareal somera. El gran tamaño de los cantos, junto con la gran dispersión de tamaños de grano y el predominio de formas angulosas, está relacionado con un escaso transporte horizontal y una baja madurez de los sedimentos (e. g. Reineck y Singh, 1980). En definitiva, estos rasgos sedimentológicos permiten inferir que el sedimento sufrió poca selección por tamaños durante el depósito y un escaso retrabajamiento por la actividad de las corrientes litorales. Es decir, una vez que se producía la descarga del sedimento en la costa, éste sufría poco movimiento por el oleaje, las mareas o las corrientes de deriva litoral, quedando en las proximidades de la desembocadura. La existencia de bases canalizadas y granoselección positiva en los paquetes conglomeráticos es congruente con esta interpretación, ya que indican una mayor influencia de los procesos fluviales sobre los litorales (Reineck y Singh, 1980). Aunque escasas, la asociación de trazas fósiles, caracterizadas por Entobia y Gastrochaenolites, también son coincidentes con un medio de depósito submareal somero (Frey y Seilacher, 1980; Bromley y Asgaard, 1993; Martinell y Domènech, 1995; Gibert et al., 1998; Gibert y Martinell, 1999).

Las propiedades tafonómicas observadas (alta fragmentación y desarticulación de los restos, presencia de facetas y concentraciones de conchas empaquetadas y encajadas) indican un medio turbulento. Estos atributos tafonómicos son congruentes con un medio somero, donde hay una alta energía hidráulica. En concreto, las concentraciones de conchas empaquetadas y encajadas se producen por la acción del oleaje de tormentas (Kidwell y Holland, 1991; Kidwell, 1991). El predominio de valvas derechas sobre valvas izquierdas de Saccostrea cuccullata (Tabla 1) también apunta hacia un medio de alta energía, ya que las valvas derechas, menos pesadas y de morfología plana, son más fácilmente transportables que las valvas izquierdas, que son fuertemente cóncavas y más pesadas. Todo esto permite interpretar las acumulaciones de fósiles estudiadas como concentraciones sedimentológicas según la nomenclatura de Kidwell et al. (1986).

No obstante, también existen evidencias que indican que el transporte sufrido por las conchas no debió ser excesivo, infiriéndose, por tanto, desplazamientos laterales pero de escasa distancia. En primer lugar están los criterios sedimentológicos expuestos anteriormente. Por otro lado, la fauna que mayoritariamente domina los niveles estudiados es de hábitats totalmente congruentes con el tipo de sedimentos en el que se encuentran. Beckvar (1988) establece esta alta correlación entre fauna y tipo de substrato como un criterio de escaso transporte en sedimentos pleistocenos de Sonora (Méjico), formados en condiciones ambientales similares a las aquí inferidas. De hecho, gasterópodos pateloides y ostreidos están entre los moluscos más abundantes en estos materiales pleistocenos.

Por otro lado, la escasez de bioperforaciones y de incrustaciones de las conchas por otros organismos indica que los restos debieron quedar expuestos sobre el substrato durante intervalos temporales cortos (e. g. Kidwell y Bosence, 1991). Este tipo de conservación se ve favorecida en condiciones ambientales como las aquí inferidas, con repetidas avalanchas de material terrígeno con una alta recurrencia. En el caso de Playa del Valle, un predominio de conchas orientadas con la concavidad hacia arriba, posición hidrodinámicamente inestable en un medio de alta energía, también puede entenderse en este contexto sedimentario. De esta forma, las conchas conservan posiciones incongruentes con las condiciones ambientales reinantes al ser rápidamente enterradas por las repetidas descargas de siliciclásticos. Así mismo, un rápido enterramiento puede usarse como un criterio adicional que indica que el transporte lateral fue de escasa magnitud, ya que los organismos fueron aislados de la zona tafonómicamente activa con relativa rapidez. 
La mezcla tafonómica de restos que indican que han sufrido desplazamientos laterales y de restos que no han sido transportados de forma sustancial nos ayuda a distinguir entre las condiciones tafonómicas reinantes durante los episodios de sedimentación normal y de eventos catastróficos ("background" y "events" sensu Speyer y Brett, 1991). Tras una avalancha de sedimento terrígeno procedente del abanico deltaico, el fondo fue colonizado por comunidades dominadas por gasterópodos pateliformes, balánidos y otros organismos típicamente litorales. El substrato debió permanecer estable durante algún tiempo, lo que permitió y favoreció que fuera colonizado. De lo contrario, el continuo desplazamiento lateral del sedimento, que tiene lugar en fondos muy inestables, inhibiría e impediría la colonización del substrato por organismos (Ziegler y Michalik, 1980; Brett y Baird, 1986; Beckvar, 1988; Aguirre y Jiménez, 1997, 1998). La alta turbulencia del medio (submareal somero) provocó que los restos, una vez muertos, fueran transportados y quedaran sometidos a procesos de desgaste, de fragmentación y de desarticulación de los elementos esqueléticos. No obstante, ocasionalmente, descargas repentinas y catastróficas de material terrígeno del abanico deltaico condicionaron el enterramiento rápido de los organismos, aislándolos de la zona tafonómicamente activa. Los atributos tafonómicos indican que, durante la mayor parte del tiempo, los fósiles permanecieron en el fondo y quedaron expuestos a los procesos destructivos típicos de los medios submareales someros. Ya que predominan los restos tafonómicamente muy alterados cabe inferir, por tanto, que la secuencia pliocena analizada es una secuencia dominada por los procesos que acontecen durante los periodos normales de la sedimentación ("background-dominated sequences" según Speyer y Brett, 1991).

Como ya indicara Meco (1977), las asociaciones de fósiles encontradas en los dos afloramientos estudiados indican unas condiciones paleoclimáticas algo más cálidas que en la actualidad debido a la existencia de organismos que tienen una distribución actual tropicalsubtropical (Tetraclita, Fisurella cf. rosea, Strombus coronatus, Nerita emiliana, Saccostrea cuccullata y Rothpletzia rudista). Por tanto, su presencia en los depósitos del Plioceno inferior de Fuerteventura sugiere la existencia de un clima más cálido durante esta época.

Numerosos estudios han confirmado la existencia de un máximo térmico a nivel global, con una temperatura media superior a la que existe actualmente, durante el Plioceno inferior (e.g. Thunell, 1979; Ruddiman et al., 1987; Zubakov, 1992). No es difícil, por tanto, pensar que Fuerteventura se situara dentro de la zona tropicalsubtropical durante este máximo térmico, cuando las bandas climáticas se desplazaron hacia latitudes más altas.

\section{INTERPRETACIÓN SECUENCIAL}

El dispositivo geométrico de solapamiento expansivo ("onlap") observado en las capas de conglomerados de la parte inferior de la secuencia, especialmente en el afloramiento de Playa del Valle, indica que el inicio de la sedimentación tuvo lugar durante una etapa transgresiva. De este modo, el primer nivel conglomerático se correspondería con el inicio de la transgresión, de tal forma que iría homogeneizando el paleorrelieve creado en el basamento (Complejo Basal), rellenando las pequeñas irregularidades erosivas existentes. Por encima, el siguiente nivel es más expansivo hacia el substrato que el inferior, lo que demuestra la progresiva subida del nivel del mar durante la etapa transgresiva. Atendiendo a los datos cronológicos obtenidos por las dataciones radiométricas existentes, dicha transgresión podría coincidir con la transgresión de la base del Plioceno.

El tramo de arenas de grano fino-medio detectado en la parte inferior-media de la secuencia de Playa del Valle (Fig. 4) podría corresponderse con los depósitos formados durante el máximo de inundación marina ("maximum flooding"). Aunque no se observan claramente las relaciones geométricas entre este tramo arenoso y los sedimentos supra e infrayacentes, existen varias evidencias que sugieren esta interpretación: 1) el nivel fosilífero de este tramo arenoso presenta un mayor contenido de fósiles con respecto a los demás niveles de acumulación. Esto podría interpretarse como un depósito formado en condiciones ligeramente más profundas; 2) este tramo arenoso se corresponde con la litología más fina de la secuencia, lo que sugiere un depósito en condiciones más profundas que los bancos conglomeráticos; 3) a su vez, supone un cambio litológico importante en la secuencia, ya que por encima se superpone un conjunto estrato y grano creciente a techo de paquetes conglomeráticos amalgamados y con bases canalizadas, que sugiere unas condiciones cada vez más someras; 4) el carácter masivo y la ausencia de estructuras sedimentarias de gran escala sugiere un depósito en un ambiente de menor energía que los supra e infrayacentes. Esta interpretación es congruente con un medio de depósito algo más profundo; finalmente, 5) el nivel fosilífero de este tramo arenoso contiene algas coralináceas formando rodolitos. Estos organismos tienen una tasa de crecimiento muy baja y, debido a su carácter fotosintético, necesitan un medio relativamente limpio, con pocas partículas de sedimento en suspensión y con un bajo aporte de particulas sedimentarias (Adey y MacIntyre, 1973; Adey y Vassar, 1975; Milliman, 1977; Bosence, 1991). El hecho de que sean organismos sensibles a la presencia de partículas sedimentarias en suspensión está en concordancia con un medio relativamente profundo, alejado de las continuas descargas de sedimentos del abanico deltaico en las zonas más someras. Estas características nos permiten inferir una tasa de sedimentación relativamente baja para este tramo arenoso, situación que suele estar asociada a los periodos de máxima inundación (Posamentier et al., 1988; Posamentier y Vail, 1988; Jervey, 1988; Vail et al., 1991; Miall, 1997).

Por encima del tramo arenoso intermedio hay un conjunto estrato creciente de bancos de conglomerados con bases erosivas y amalgamados. Este conjunto podría interpretarse como los depósitos formados durante el cortejo sedimentario de alto nivel del mar ("highstand 


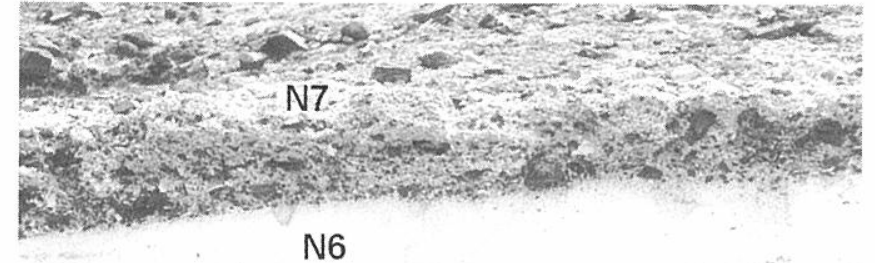

N6

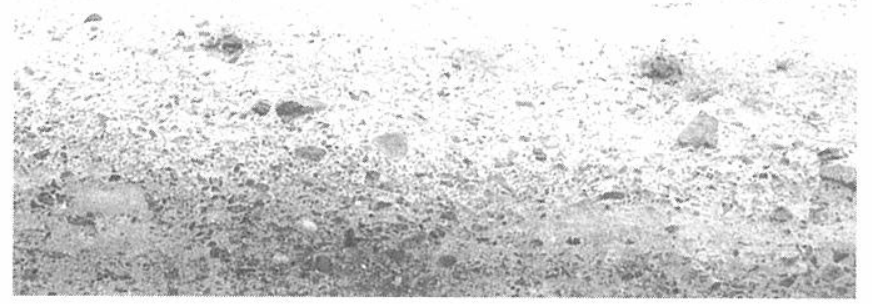

Figura 4. Detalle del nivel de arenas presente en la mitad inferior de la sección Playa del Valle en el que se pueden observar los niveles fosilíferos 6 y 7 ( N-6 y N-7 en la Fig. 2). Estos sedimentos se interpretan como los depósitos formados durante las condiciones de máxima inundación ("maximum flooding deposits").

Detail of the sand deposit in the lower part of the Playa del Valle section with the fossiliferous beds 6 and 7 (N6 and N7 in Fig. 2). These sediments are interpreted as the maximun flooding deposits.

systems tract") por la progradación del abanico deltaico. Este dispositivo indica una progresiva somerización de las facies y, por tanto, un mayor acercamiento a la desembocadura del sistema fluvial que generó el abanico deltaico. De ahí, que los bancos conglomeráticos muestren cada vez mayor espesor, más superficies de amalgamación y superficies erosivas más netas.

En definitiva, la secuencia pliocena de Playa del Valle representa un ciclo sedimentario completo de profundización-somerización formado, probablemente, durante un ascenso y descenso eustático del nivel del mar. En el afloramiento Barranco León las relaciones estratigráficas de los sedimentos no están tan bien expuestas como en Playa del Valle, de ahí que sea más difícil una interpretación secuencial de esta sección. Aquí, lo que se pone claramente de manifiesto es la somerización final del conjunto plioceno estudiado por la superposición de depósitos de arenas eólicas sobre los conglomerados fosilíferos de la base.

\section{CONCLUSIONES}

Los materiales que constituyen las secciones pliocenas estudiadas en la isla de Fuerteventura se depositaron en un ambiente de abanico deltaico, en zonas próximas a la costa, y apenas experimentaron modificaciones debidas a procesos marinos, como oleaje, corrientes, etc.

La fauna que contienen se corresponde con la de un ambiente litoral somero, constituida por formas pateloides, ostreidos y cirrípedos, corroborado también por la asociación de trazas fósiles, caracterizada por Entobia y Gastrochaenolites. Las alteraciones tafonómicas que presentan estos fósiles (alta fragmentación o presencia de facetas) están en concordancia con un medio de alta energía hidraúlica, como es en el caso de un medio litoral poco profundo. Esto permite interpretar las acumulaciones de fósiles estudiadas como concentraciones sedimentológicas según Kidwell et al. (1986). El transporte en la horizontal de estos fósiles, sin embargo, no debió de ser importante, ya que se produjeron enterramientos catastróficos repentinos como consecuencia de los continuos aportes de material siliclástico desde el abanico deltaico. Esto es coherente con el tipo de fauna y de substrato, la escasez de bioperforaciones, el escaso redondeamiento de los cantos y la pobre selección por tamaños, y el que la mayoría de las conchas se encuentran con la concavidad hacia arriba. Por tanto, la predominancia de restos fósiles muy alterados tafonómicamente permite inferir que se trata de una secuencia dominada por los procesos que acontecen durante los períodos normales de la sedimentación ("background-dominated sequences" según Speyer y Brett, 1991).

La existencia en estos depósitos pliocenos de algunas especies que tienen una distribución tropical-subtropical actualmente, permite inferir la hipótesis de un desplazamiento latitudinal hacia el norte de las bandas tropicales durante este período de tiempo, en condiciones globales de temperaturas más elevadas.

Según el dispositivo geométrico de solapamiento expansivo ("onlap") que se observa en las capas conglomeráticas de la parte inferior de la secuencia, el inicio de la sedimentación se produjo durante una etapa transgresiva. El tramo de arenas observado en la parte inferior-media de la secuencia de Playa del Valle podría corresponderse con los depósitos formados durante el máximo de inundación marina ("maximum flooding"). El conjunto estrato creciente de bancos de conglomerados con bases erosivas y amalgamados, situado por encima del tramo arenoso, podría interpretarse como los formados durante el alto nivel del mar ("higstand-system tract"). Por lo tanto, la secuencia pliocena de Playa del Valle representa un ciclo sedimentario completo de profundización-somerización formado, probablemente, durante un ascenso y descenso eustático del nivel del mar.

\section{AGRADECIMIENTOS}

Julio Aguirre agradece al Proyecto de Investigación "Cambios climáticos en el sur de España durante el Neógeno" de la Fundación Ramón Areces la ayuda prestada para la realización de este trabajo. Los autores agradecen al Dr. Sixto Fernández López sus numerosos comentarios en el campo y durante la preparación del manuscrito. Vaya nuestro especial agradecimiento también a los Drs. Usera, González Delgado y Martinell, revisores de este trabajo, cuyas sugerencias y anotaciones han mejorado sustancialmente la calidad del mismo. 


\section{BIBLIOGRAFÍA}

Adey, W.H. and MacIntyre, I.G. 1973. Crustose coralline algae: A re-evaluation in the geological sciences. Geological Society of America Bulletin, 84, 883-904.

Adey, W.H. and Vassar, J.M. 1975. Colonization, succession and growth rates of tropical crustose coralline algae (Rhodophyta, Cryptonemiales). Phycologia, 14, 55-69.

Aguirre, J. 1998. Bioconstrucciones de Saccostrea cuccullata Born, 1778 en el Plioceno superior de Cádiz (SO de España): implicaciones paleoambientales y paleoclimáticas. Revista Española de Paleontología, 13, 27-36.

Aguirre, J. y Jiménez, A.P. 1994. ¿Por qué Saccostrea cuccullata Born, 1778 crece como crece y donde crece? X Jornadas de Paleontologİa, Madrid,1-3.

Aguirre, J. and Jiménez, A.P. 1997. Census assemblages in hard-bottom coastal communities: A case study from the Plio-Pleistocene Mediterranean. Palaios, 12, 598-608.

Aguirre, J. and Jiménez, A.P. 1998. Fossil analogues of present-day Cladocora caespitosa coral banks: Sedimentary setting, dwelling community, and taphonomy (Late Pliocene, W Mediterranean). Coral Reefs, 17, 203-213.

Beckvar, N. 1988. Minimal shell transport detected in a coarse-grained Pleistocene marine terrace, Sonora. Palaios, 3, 365-369.

Beesley, P.L., Ross, G.J.B. and Wells, A. (Eds.) 1998. Mollusca: the Southern Synthesis. Fauna of Australia. Vol. 5. CSIRO Publishing. Melbourne, $1.234 \mathrm{pp}$.

Bosence, D.W.J. 1991. Coralline algae: Mineralization, taxonomy, and palaeoecology. In: Calcareous Algae and Stromatolites (Ed. R. Riding). Springer Verlag, BerlÌn, 98-113.

Brett, C.E. and Baird, G.C. 1986. Comparative taphonomy: A key to paleoenvironmental interpretation based on fossil preservation. Palaios, 1, 207-227.

Bromley, R.G. and Asgaard, U. 1993. Two bioerosion ichnofacies produced by early and late burial associated with sea-level change. Geologische Rundschau, 82, 276280.

Coello, J., Cantagrel, J.M., Hernán, F., Fúster, J.M., Ibarrola, E., Ancochea, E., Casquet, C., Jamond, C., Díaz de Terán, J.R. and Cendrero, A. 1992. Evolution of the eastern volcanic ridge of the Canary Islands based on new K-Ar data. Journal of Volcanology Geothermal Research, 53, 251-274.

Fúster, J.M., Cendrero, A., Gastesi, P., Ibarrola, A. y López Ruiz, J. 1968. Geología y volcanismo de las Islas Canarias, Fuerteventura. Instituto Lucas Mallada, CSIC, Madrid, 239 pp.

Frey, R.W. and Seilacher, A. 1980. Uniformity in marine invertebrate ichnology. Lethaia, 13, 183-207.

Gibert, J.M. de and Martinell, J. 1999. Proximal-distal variations of trace fossil assemblages in a Pliocene ria, Baix Llobregat, northeastern Spain. Revista de la Sociedad Geológica de España, 12, 209-214.

Gibert, J.M. de, Martinell, J. y Domènech, R. 1996. El Mioceno marino entre las playas de L'Arrabassada y El Miracle (Tarragona): aspectos paleontológicos e implicaciones sedimentológicas. Acta Geológica Hispánica, 29, 133-148.

Gibert, J.M. de, Martinell, J. and Domènech, R. 1998. Entobia ichnofacies in fossil rocky shores, Lower Pliocene, northwestern Mediterranean. Palaios, 13, 476487.

Harry, H.W. 1985. Synopsis of the supraspecific classification of living oysters (Bivalvia: Gryphaeidae and Ostreidae). The Veliger, 28, 121-158.

Jervey, M.T. 1988. Quantitative geological modeling of siliciclastic rock sequences and their seismic expression. In: Sea-Level Changes: An Integrated Approach (Eds. C.K. Wilgus, B.S. Hastings, C.G.St.C. Kendal, H.W. Posamentier, C.A. Ross \& C. Van Wagoner). Society of Economic Paleontologists and Mineralogists, Special Publication, 42, 47-69.

Johnson, M.E. 1988. Why are ancient rocky shores so uncommon?. Journal of Geology, 96, 469-480.

Johnson, M.E. 1992. Studies on ancient rocky shores: A brief history and annotated bibliography. Journal of Coastal Research, 8, 797-812.

Johnson, M.E. and Baarli, G. 1999. Diversification of rockyshore biotas through geologic time. Geobios, 32, 257273.

Kidwell, S.M. 1991. The stratigraphy of shell concentrations. In: Taphonomy. Releasing the Data Locked in the Fossil Record (Eds. P.A. Allison, y D.E.G. Briggs). Plenum Press, New York, 211-290.

Kidwell, S.M. and Bosence, D.W.J. 1991. Taphonomy and time-averaging of marine shelly faunas: In: Taphonomy. Releasing the Data Locked in the Fossil Record (Eds. P.A. Allison \& D.E.G. Briggs). Plenum Press, New York, 115-209.

Kidwell, S.M. and Holland, S.M. 1991. Fields description of coarse bioclastic fabrics. Palaios, 6, 426-434.

Kidwell, S.M., Fürsich, F.T. and Aigner, T. 1986. Conceptual framework for the analysis and classification of fossil concentrations. Palaios, 1, 228-238.

Martinell, J. and Domènech, R. 1995. Bioerosive structures on the Pliocene rocky shores of Catalonia (Spain). Revista Española de Paleontologİa, 10, 37-44.

Meco, J. 1975. Los niveles con Strombus de JandÌa (Fuerteventura, Islas Canarias). Anuario de Estudios Atlánticos, 21, 643-660.

Meco, J. 1977. Paleontología de Canarias. Los Strombus Neógenos y Cuaternarios del Atlántico Euroafricano (Taxonomía, Bioestratigrafía y Paleoecología). Tomo I. Excmo. Cabildo Insular de Gran Canaria, 142 pp., 31 láms.

Meco, J. and Stearns, C.E. 1981. Emergent littoral deposits in the Eastern Canary Islands. Quaternary Research, 15, 199-208.

Miall, A.D. 1997. The Geology of Stratigraphic Sequences. Springer Verlag, Berlín, 433 pp.

Milliman, J.D. 1977. Role of calcareous algae in atlantic continental margin sedimentation. In: Fossil Algae, Recent Results and Developments (Ed. E. Flügel). Springer Verlag, Berlín, 232-247.

Morton, B. and Chan, K.Y. 1986. The salinity tolerances of four species of bivalves from Hong Kong mangrove. In: 
The Marine Flora and Fauna of Hong Kong and Southern China (Ed. B. Morton). Proceedings of the 2nd International Marine Biological Workshop, Hong Kong University Press, 1115-1121.

Okemwa, E., Ruwa, R.K. and Polk, P. 1986. The autoecology of the edible oyster Crassostrea cucullata Born, 1778: Size related vertical distribution at Mkomani, Mombasa, Kenya. Journal of Science Serie (B), 7, 9-14.

Posamentier, H.W. and Vail, P.R. 1988. Eustatic controls on clastic deposition. II-Sequence and systems tract models. In: Sea-Level Changes: An Integrated Approach (Eds. C.K. Wilgus, B.S. Hastings, C.G.St.C. Kendal, H.W. Posamentier, C.A. Ross y C. Van Wagoner). Society of Economic Paleontologists and Mineralogists Special Publication, 42, 125-154.

Posamentier, H.W., Jervey, M.T. and Vail P.R. 1988. Eustatic controls on clastic deposition. I-Conceptual framework. In: Sea-Level Changes: An Integrated Approach (Eds. C.K. Wilgus, B.S. Hastings, C.G.St.C. Kendal, H.W. Posamentier, C.A. Ross \& C. Van Wagoner). Society of Economic Paleontologists and Mineralogists Special Publication, 42, 109-124.

Powell, E.N., Staff, G.M., Davies, D.J. and Callender, W.R. 1989. Macrobenthic death assemblages in modern marine environments: formation, interpretation and aplication. Aquatic Science, 1, 555-589.

Reineck, H.E. and Singh, I.B. 1980. Depositional sedimentary enviroments. With reference to terrigenous clastics. (2nd edition). Springer Verlag, Berlín, 550 pp.

Ruddiman, W.F., Backman, J., Baldauf, J., Hooper, P., Keigwin, K., Miller, K., Raymo, M. and Thomas, E. 1987. Leg 94 paleoenvironmental synthesis. Initial Reports of the Deep Sea Drilling Project, 94, 1207-1215.

Silva, C.M. da, Cachao, M., Martinell, J. and Domènech, R. 1999. Bioerosional evidence of rocky paleoshores in the
Neogene of Portugal: environmental and stratigraphical ssignificance. Bulletin of the Geological Society of Denmark, 45, 156-160.

Speyer, S.E. and Brett, C.E. 1991. Taphofacies controls: Background and episodic processes in fossil assemblage preservation. In: Taphonomy. Releasing the Data Locked in the Fossil Record (Eds. P.A. Allison \& D.E.G. Briggs). Plenum Press, New York, 502-545.

Stenzel, H.B. 1971. Oysters. In: Treatise on Invertebrate Paleontology. Mollusca 6. Bivalvia, Part N (Ed. R.C. Moore). The Geological Society of America and the University of Kansas Press, 3, 954-1224.

Thunell, R.C. 1979. Climatic evolution of the Mediterranean Sea during the last 5.0 million years. Sedimentary Geology, 23, 67-79.

Vail, P.R., Audemard, F., Bowman, S.A., Eisner, P.N. and Perez-Cruz, C. 1991. The stratigraphic signatures of tectonics, eustasy and sedimentology. In: Cycles and Events in Stratigraphy (Eds. G. Einsele, W. Ricken \& A. Seilacher). Springer Verlag, Berlín, 617-659.

Ziegler, M. and Michalik, J. 1980. Later Triassic serpulids (Annelida, Polychaetia, Sedentaria) in the western Carpathians. Geologicky Zbornick-Geologica Carpathica, 31, 621-640.

Zubakov, V. 1992. Correlation of the late Neogene climate events of Atlantic, Mediterranean and Ponto-Caspian. 1st. Congress R.C.A.N.S., Lisboa, 119-122.

Zullo, V.A. 1986. Quaternary barnacles from the Gal-pagos Islands. Proceedings of the California Academy of Sciences, 44, 55-66.

Zullo, V.A. 1991. Zoogeography of the shallow-water cirriped fauna of the Galápagos Islands and adjacent regions in the tropical eastern Pacific. In: Galápagos Marine Invertebrates. Taxonomy, Biogeography, and Evolution in Darwin's Islands (Ed. M.J. James). Plenum Publishing Company, New York, 173-192. 